05,11

\title{
Одноионный механизм слабого антиферромагнетизма и спин-флоп-переход в двухподрешеточном ферромагнетике
}

\author{
(c) С.Н. Мартынов \\ Институт физики им. Л.В. Киренского ФИЦ КНЦ СО РАН, \\ Красноярск, Россия \\ E-mail: unonav@iph.krasn.ru \\ Поступила в Редакцию 31 января 2020 г. \\ В окончательной редакции 31 января 2020 г. \\ Принята к публикации 4 фревраля 2020 г.
}

\begin{abstract}
Основное состояние гейзенберговского ферромагнетика с неколлинеарными осями одноионной анизотропии двух магнитных подрешеток исследовано во внешнем магнитном поле, приложенном в плоскости содержащей оси анизотропии. Неколлинеарность локальных осей анизотропии подрешеток приводит к новому эффекту - ориентационному фазовому переходу первого рода типа спин-флоп. Поле перехода зависит от величины одноионной анизотропии и ориентации локальных осей подрешеток. Анализ устойчивости магнитных состояний показывает, что переход сопровождается гистерезисом в полевой зависимости намагниченности. Определены зависимости поля спин-флоп-перехода, величины скачка намагниченности и восприимчивости от величины одноионной анизотропии и ориентации ее осей. Полученные результаты использованы для объяснения полевой зависимости намагниченности в ферромагнитном кристалле $\mathrm{PbMnBO}_{4}$.
\end{abstract}

Ключевые слова: ферромагнетизм. одноионная анизотропия, ориентационный фазовый переход.

DOI: 10.21883/FTT.2020.07.49468.017

\section{1. Введение}

Термин „слабый антиферромагнетизм“ (weak antiferromagnetism) был введен Вонсовским и Туровым [1-3] по аналогии со слабым ферромагнетизмом для определения магнитного упорядочения в магнетике с основным ферромагнитным обменом и неколлинеарностью подрешеток, вызванной релятивистским обменом Дзялошинского-Мория (DM) [4,5]

$$
H_{D M}=\mathbf{D}\left[\mathbf{M}_{1} \times \mathbf{M}_{2}\right],
$$

где $M_{1}$ и $M_{2}$ - магнитные моменты подрешеток. При этом типе упорядочения абсолютная величина вектора антиферромагнетизма $l$ мала по сравнению с полным магнитным моментом $m$

$$
|\mathbf{I}|=\left|\mathbf{M}_{1}-\mathbf{M}_{2}\right| \ll\left|\mathbf{M}_{1}+\mathbf{M}_{2}\right|=|\mathbf{m}| .
$$

Слабый антиферромагнетизм, вызванный взаимодействием DM, наблюдался, например, в соизмеримой магнитной фазе кристалла $\mathrm{MnSi}$ [6] и в одной из антиферромагнитных подрешеток ионов меди в $\mathrm{Ba}_{3} \mathrm{Cu}_{2} \mathrm{O}_{4} \mathrm{Cl}_{2}$ [7].

Другой причиной скоса магнитных моментов подрешеток является неколлинеарность локальных осей одноионной анизотропии (ОА) в магнетиках с несколькими магнитными ионами в элементарной ячейке кристалла. Впервые такой одноионный механизм неколлинеарности в антиферромагнетике был рассмотрен Мория для описания слабого ферромагнетизма в антиферромагнитном кристалле $\mathrm{NiF}_{2}$ со структурой рутила [8] (см. также краткое изложение этого анализа в книге [9]). В этом магнетике легкие оси ОА, расположенные в плоскости $a b$ кристалла, взаимно ортогональны. Условия появления неколлинеарности, вызванной одноионной анизотропией, были рассмотрены при симметрийном анализе орторомбических пировскитов в работе [10]. Неколлинеарность локальных осей ОА в значительной степени определяет магнитные свойства ферритов [11]. При развороте легких осей подрешеток относительно общей легкой оси кристалла коллинеарное упорядочение становится неустойчивым относительно отклонений моментов от общего легкого направления из-за появления линейного по углу члена в разложении энергии анизотропии

$$
\epsilon_{a, i} \propto K_{i} \cos ^{n}\left(\theta_{i}-\theta_{K i}\right)
$$

Здесь $n-$ порядок анизотропии, $\theta_{i}-$ угол ориентации момента $i$ и $\theta_{K i}$ - направление оси анизотропии этого момента. При условии неколлинеарности осей ОА моментов $\theta_{K i} \neq \theta_{K j}$ такая неустойчивость, как и в случае взаимодействия DM, является абсолютной - неколлинеарность возникает при любом значении параметра $K_{i}$. Принципиальным отличием одноионного механизма неколлинеарности от взаимодействия DM является его анизотропный характер. Энергии ОА зависит от ориентации моментов относительно кристаллических осей, в то время как энергия взаимодействия DM изотропна при одновременном повороте моментов в плоскости ортогональной вектору D. При низкосимметричном окружении магнитных ионов со спином $S>1 / 2$ слабый ферромагнитный момент формируется как одноионным, так и двухионным (в частности взаимодействием DM) механизмами анизотропии. Это проявляется, прежде всего, 
в различной зависимости кривых намагниченности вдоль разных кристаллических осей. При этом конкурирующая OA в зависимости от ориентации магнитного поля может либо увеличивать величину слабого момента либо ее уменьшать [12-15]. Ориентационный спин-флоппереход между состояниями с различной ориентацией моментов в антиферромагнетике может стать фазовым переходом первого рода [16]. Вывод о доминирующем влияние ОА на формирование слабого ферромагнитного момента был сделан также для $\mathrm{K}_{2} \mathrm{NiF}_{4}, \mathrm{La}_{2} \mathrm{NiO}_{4}$, $\mathrm{La}_{2} \mathrm{CoO}_{4}[13], \mathrm{LaMnO}_{3}[17,18]$.

На настоящий момент информация о неколлинеарности магнитных моментов, вызванной конкуренцией ОА кристаллографически эквивалентных подрешеток в неметаллических ферромагнетиках, отсутствует. При магнитных измерениях обнаружить существование слабого антиферромагнитного момента на фоне большой общей намагниченности сложно. Присутствие такого момента должно проявляться при переориентации в магнитном поле, приложенном вдоль вектора слабого антиферромагнетизма - спин-флоп-переходе. При этом продольная намагниченность меняется скачком. Сильно анизотропный характер кривых намагниченности ферромагнитного кристалла $\mathrm{PbMnBO}_{4}$ [19] со скачком магнитного момента в поле, приложенном вдоль орторомбической оси $b$, дает основание сделать предположение о доминирующей роли одноионного механизма неколлинеарности в этом магнетике.

Целью настоящей работы является теоретическое описание ориентационного фазового перехода в рамках простой модели ферромагнетика с неколлинеарными осями анизотропии двух подрешеток и определение зависимости наблюдаемых магнитных параметров от параметров локальной анизотропии подрешеток.

\section{2. Модель и решения}

Рассмотрим основное состояние однопозиционного магнетика с плоскостью симметрии кристалла m (или поворотной осью второго порядка $C_{2}$ ) между трансляционно неэквивалентными позициями магнитных ионов. Эти условия типичны для магнитных кристаллов ряда групп наиболее многочисленного класса ромбических кристаллов, в частности, $\mathrm{PbMnBO}_{4}$ [19-21]. Гамильтониан системы спинов с локальными осями двухосной ОА $z 1, x 1$ и $z 2, x 2$ двух магнитных подрешеток может быть записан в виде

$$
\begin{aligned}
H & =J \sum_{i j} \mathbf{S}_{i} \mathbf{S}_{j}+K_{1}\left(\sum_{i} S_{i}^{z 1^{2}}+\sum_{j} S_{j}^{z 2^{2}}\right) \\
& +K_{2}\left(\sum_{i} S_{i}^{x 1^{2}}+\sum_{j} S_{j}^{x 2^{2}}\right)+g \mu_{B} \mathbf{H}_{0}\left(\sum_{i} \mathbf{S}_{i}+\sum_{j} \mathbf{S}_{j}\right)
\end{aligned}
$$

Выберем оси $z_{1}$ и $z_{2}$ в качестве легких осей намагничивания в каждой подрешетке $K_{1}<0$. Знак второй
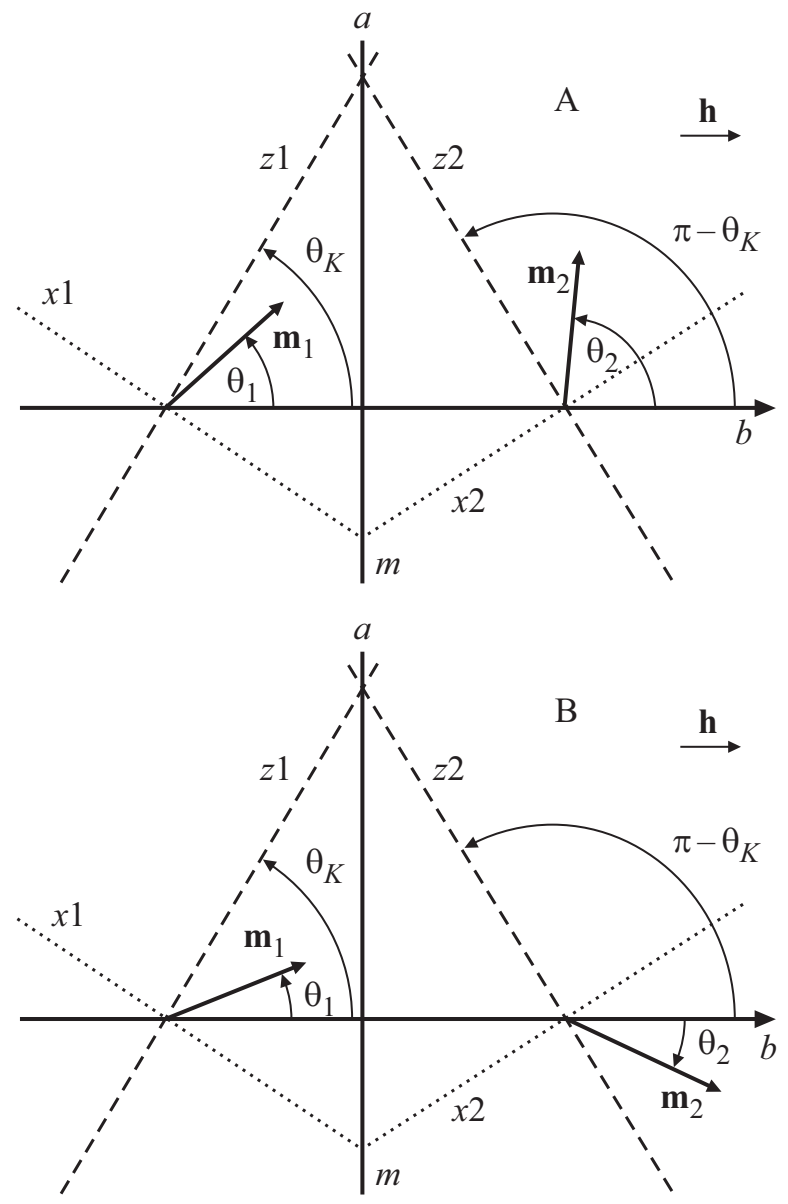

Рис. 1. Ориентация моментов ферромагнитных подрешеток $m_{1}$ и $m_{2}$ в двух различных состояниях. При угле между легкими осями подрешеток $\theta_{K}>\pi / 4$ и $h<h_{s f}$ основным является состояние А, при $h>h_{s f}-$ состояние В.

константы локальной анизотропии $K_{2}$ (оси $x_{1}$ и $x_{2}$ ) может быть любой. Нетрудно показать, что при изотропном ферромагнитном обмене $J<0$ моменты всегда будут лежать в плоскости, образованной направлениями легкой оси и внешнего магнитного поля. Для случая внешнего магнитного поля, приложенного в плоскости ортогональной плоскости симметрии $\mathbf{m}$ и содержащей локальные оси анизотропии $K_{1}, K_{2}$ моментов подрешеток $i$ и $j$, получаем компланарную задачу (рис. 1). Это существенно упрощает ее решение, сводя его к нахождению двух углов ориентации моментов подрешеток $\theta_{1}$ и $\theta_{2}$ относительно направления внешнего магнитного поля.

Ортогональность локальных осей анизотропии в каждой подрешетке и ограничения симметрии

$$
\theta_{x 1}=\theta_{z 1}+\pi / 2, \quad \theta_{z 2}=\pi-\theta_{z 1}, \quad \theta_{x 2}=\pi-\theta_{x 1}
$$

позволяют выразить энергию анизотропии через один эффективный параметр анизотропии $K=K_{1}-K_{2}$ и угол легкой оси $\theta_{K}=\theta_{z 1}$. Энергия основного состояния 
классических моментов $\mathbf{m}_{1,2}=-g \mu_{B} \mathbf{S}_{1,2}$ во введенных переменных и обозначениях (рис. 1) имеет вид

$$
E=\frac{N}{2}|J| S^{2} z \cdot \epsilon\left(\theta_{1}, \theta_{2}\right),
$$

где $z$ - число магнитных соседей,

$$
\begin{aligned}
\epsilon\left(\theta_{1}, \theta_{2}\right)= & -\cos \left(\theta_{1}-\theta_{2}\right)+a\left(\cos ^{2}\left(\theta_{1}-\theta_{K}\right)\right. \\
& \left.+\cos ^{2}\left(\theta_{2}+\theta_{K}\right)\right)-h\left(\cos \theta_{1}+\cos \theta_{2}\right), \\
a= & \frac{K}{|J| z}<0, \quad h=\frac{g \mu_{B} H_{0}}{|J| S z}>0
\end{aligned}
$$

- нормированные энергия, величина локальной анизотропии и внешнее магнитное поле, соответственно. Минимизация нормированной энергии $\epsilon\left(\theta_{1}, \theta_{2}\right)$ приводит к системе из двух уравнений

$$
\begin{aligned}
\sin \left(\theta_{1}-\theta_{2}\right) & +h \sin \theta_{1} \\
& -2 a \cdot \cos \left(\theta_{1}-\theta_{K}\right) \sin \left(\theta_{1}-\theta_{K}\right)=0 \\
-\sin \left(\theta_{1}-\theta_{2}\right) & +h \sin \theta_{2} \\
& -2 a \cdot \cos \left(\theta_{2}+\theta_{K}\right) \sin \left(\theta_{2}+\theta_{K}\right)=0,
\end{aligned}
$$

которая может быть преобразована в систему уравнений на сумму и разность углов ориентации моментов

$$
\begin{aligned}
& h \cdot \sin \frac{\theta_{1}+\theta_{2}}{2} \cos \frac{\theta_{1}-\theta_{2}}{2} \\
&-a \cdot \sin \left(\theta_{1}+\theta_{2}\right) \cos \left(\theta_{1}-\theta_{2}-2 \theta_{K}\right)=0 \\
& \sin \left(\theta_{1}-\theta_{2}\right)+h \sin \frac{\theta_{1}-\theta_{2}}{2} \cos \frac{\theta_{1}+\theta_{2}}{2} \\
& \quad-a \cdot \cos \left(\theta_{1}+\theta_{2}\right) \sin \left(\theta_{1}-\theta_{2}-2 \theta_{K}\right)=0 .
\end{aligned}
$$

Решения системы (3) определяют два возможных состояния - магнитные фазы А и В (рис. 1):

$$
\begin{aligned}
& A: \quad \cos \frac{\theta_{1}+\theta_{2}}{2}=\frac{h \cos \left(\left(\theta_{1}-\theta_{2}\right) / 2\right)}{2 a \cos \left(\theta_{1}-\theta_{2}-2 \theta_{K}\right)}, \\
& B: \theta_{1}=-\theta_{2} .
\end{aligned}
$$

Первое решение описывает несимметричную ориентацию моментов подрешеток относительно магнитного поля (оси $b$ ), второе - ориентацию симметричную. В отсутствии внешнего магнитного поля значения переменных и энергии состояний определяются равенствами

$$
h=0
$$

$A: \tan \delta_{0}=-\frac{a \cdot \sin 2 \theta_{K}}{1+a \cdot \cos 2 \theta_{K}}, \quad \delta_{0}=\theta_{2}-\left.\theta_{1}\right|_{h=0}$,

$$
\epsilon_{A 0}=-\sqrt{1+a^{2}+2 a \cdot \cos 2 \theta_{K}}
$$

$B: \tan 2 \theta_{0}=-\frac{a \cdot \sin 2 \theta_{K}}{1-a \cdot \cos 2 \theta_{K}}, \quad \theta_{0}=\theta_{h=0}$, $\epsilon_{B 0}=-\sqrt{1+a^{2}-2 a \cdot \cos 2 \theta_{K}}$.
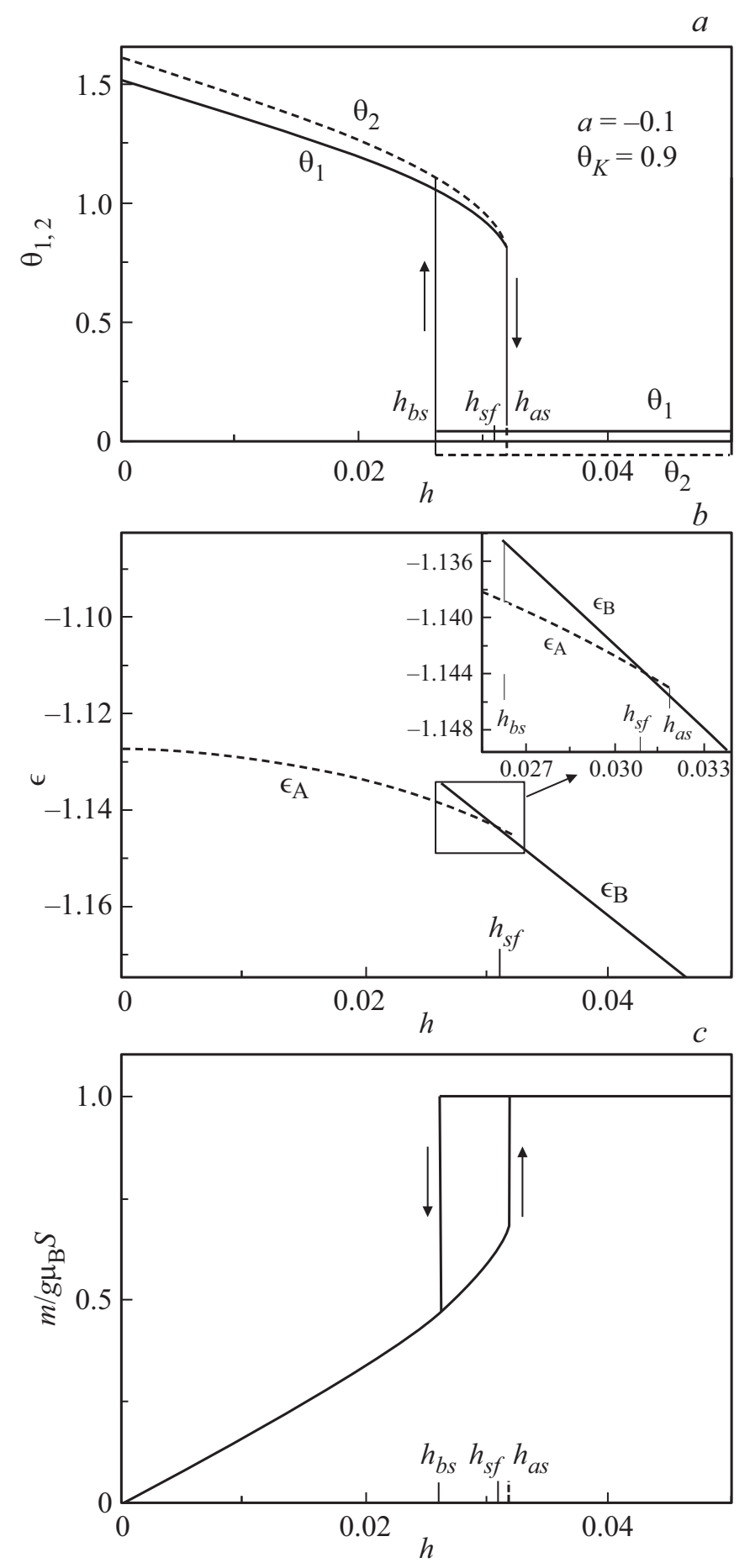

Рис. 2. Полевые зависимости $a$ ) углов ориентации магнитных моментов подрешеток $\left.\theta_{1}, \theta_{2}, b\right)$ энергии фаз А и В и c) проекции намагниченности на направление внешнего поля при относительной величине локальной анизотропии $a=-0.1$ и угле ориентации легкой оси анизотропии $\theta_{K}=0.9$. На вставке $b$ ) увеличена область пересечения энергий фаз $\epsilon_{\mathrm{A}}$ и $\epsilon_{\mathrm{B}}$. Вертикальные стрелки показывают направление изменения значений углов $\theta_{1,2}$, энергии и продольной намагниченности на границах устойчивости фаз А и В $h_{a s}$ и $h_{b s}$, соответственно. $h_{s f}$ - поле, при котором энергии фаз совпадают. 


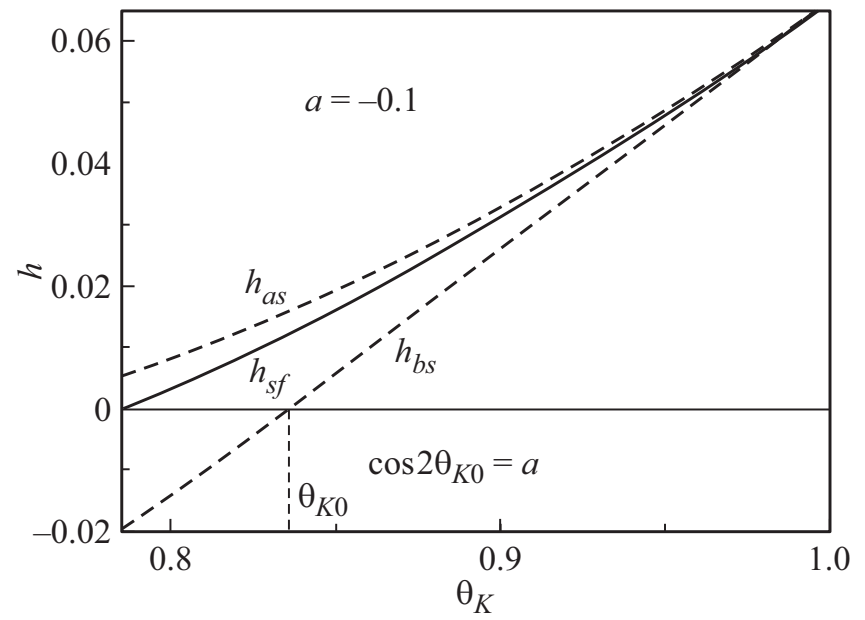

Рис. 3. Зависимость границ устойчивости фаз А $\left(h_{a s}\right)$ и В $\left(h_{b s}\right)$ (штриховые линии) от угла ориентации оси анизотропии $\theta_{K}$ при параметре анизотропии $a=-0.1$.

Следовательно, при выполнении условий

$$
a \cdot \cos 2 \theta_{K}>0, \quad \Rightarrow a<0, \quad \theta_{K}>\pi / 4
$$

$\epsilon_{A 0}<\epsilon_{B 0}-$ нижним по энергии состоянием является фаза А с ориентацией средней намагниченности вдоль оси $a$ (рис. 1). При $\theta_{K}<\pi / 4$ общим легким направлением становится ось $b$, и начиная с нулевого поля реализуется фаза В.

Рассмотрим изменение ориентации магнитных моментов при выполнении условия (5) в магнитном поле, приложенном вдоль трудной оси $b$. С ростом величины магнитного поля угол $\theta_{2}$ меняется быстрее угла $\theta_{1}$ и в поле $h=\sqrt{2} a \cos 2 \theta_{K}$ моменты ориентируются коллинеарно $\left(\theta_{1}=\theta_{2}=\pi / 4\right)$. Дальнейшее увеличение поля может изменить знак $\delta=\theta_{2}-\theta_{1}$. Однако еще раньше могут сравняться энергии фаз. При этом значении поля $h_{s f}=h\left(\epsilon_{\mathrm{A}}=\epsilon_{\mathrm{B}}\right)$ произойдет изменение основного состояния - фазовый переход по магнитному полю. В зависимости от величины локальной анизотропии $a$ и угла ориентации легких осей $\theta_{K}$ переход между фазами А и В может быть переходом как первого, так и второго рода. Эволюция основного состояния во внешнем поле для $a=-0.1$ и $\theta_{K}=0.9$ показана на рис. 2. Рассмотренный ориентационный фазовый переход имеет очевидную аналогию со спин-флоп-переходом в обычном антиферромагнетике. При $h \rightarrow 0$ поле направлено вдоль вектора слабого антиферромагнетизма $\mathbf{l}=\mathbf{m}_{1}-\mathbf{m}_{2}$. После перехода вектор $\mathbf{l}$ ориентирован ортогонально внешнему полю (рис. 1).

При существовании различных решений рассматриваемой модели важно определить область устойчивости каждого состояния. В случае фазового перехода первого рода это дает информацию о величине возможного гистерезиса намагниченности. Области устойчивости решений (4) определяются положительной определенно- стью главных миноров определителя [22]

$$
\left\|\partial^{2} \epsilon / \partial \theta_{n} \partial \theta_{m}\right\|, \quad(n, m=1,2) .
$$

При $|a|, h \ll 1, \quad \partial^{2} \epsilon / \partial \theta_{1,2}^{2}>0$ и уравнение

$$
\frac{\partial^{2} \epsilon}{\partial \theta_{1}^{2}} \cdot \frac{\partial^{2} \epsilon}{\partial \theta_{2}^{2}}-\frac{\partial^{2} \epsilon}{\partial \theta_{1} \partial \theta_{2}} \cdot \frac{\partial^{2} \epsilon}{\partial \theta_{2} \partial \theta_{1}}=0,
$$

совместно с уравнениями минимизации энергии (2) для каждой фазы (4), определяет границы устойчивости фаз $h_{a s}, h_{b s}$. Перекрывание областей устойчивости определяет интервал полей в котором возможен гистерезис кривых намагниченности. Этот интервал существенно зависит от угла ориентации оси анизотропии $\theta_{K}$ и для $a=-0.1$. обращается в ноль при $\theta_{K} \approx 1.01$ (рис. 3 ). Границы совпадают со значением поля перехода $h_{s f}$, что соответствует фазовому переходу второго рода. Изменение типа фазового перехода обсуждается в разделе 4. При развороте осей анизотропии близком к $\pi / 4\left(\theta_{K}<\theta_{K 0}=0.5 \arccos a\right)$ фаза В устойчива при любом значении магнитного поля (граница устойчивости $\left.h_{b s}<0\right)$.

\section{3. Восприимчивость}

Отличительной чертой каждого типа магнитного упорядочения является зависимость магнитного момента от величины и направления приложенного поля. Как правило, измеряется проекция намагниченности на направление приложенного поля - продольная намагниченность. И хотя в случае сложной, в частности, неколлинеарной магнитной структуры такая информация является неполной, она отражает интегральные анизотропные свойства магнетика. Начальный участок полевой зависимости продольной намагниченности в поле, приложенном вдоль трудного направления магнитного кристалла,

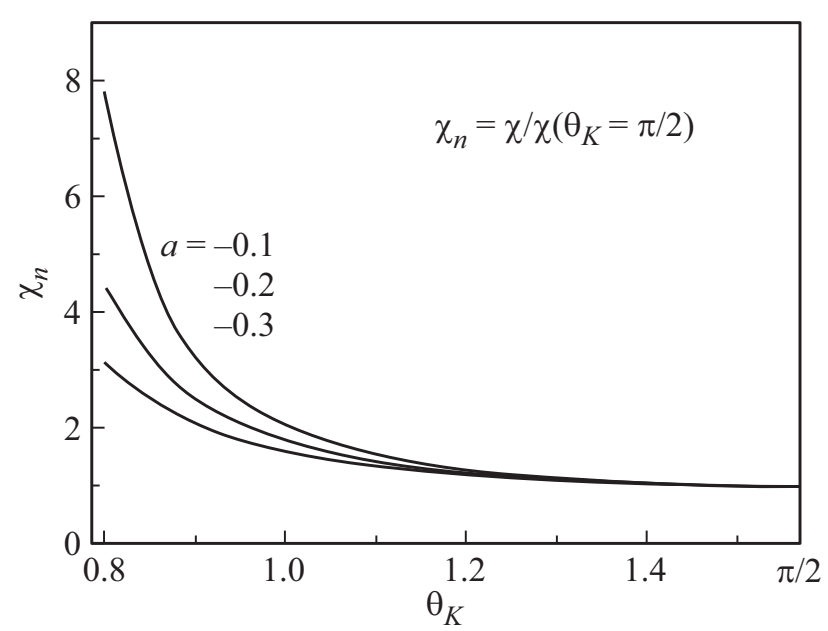

Рис. 4. Зависимость начальной восприимчивости, нормированной на восприимчивость коллинеарного ферромагнетика $\left(\chi\left(\theta_{K}=\pi / 2\right)\right)$, от угла ориентации осей анизотропии $\theta_{K}$ при различных значениях параметра анизотропии $a$. 
несет информацию о величине магнитной анизотропии, что позволяет в случае коллинеарного ферромагнетика определить ее значение уже в сравнительно малых полях. Однако в случае неколлинеарных локальных осей подрешеток мы имеем дело с некоторой эффективной анизотропией, усредненной по локальным значениям. Это дает значение общей анизотропии всего магнетика меньшее локальных значений [11]. Как следствие, наклон кривых намагниченности (начальная восприимчивость) может значительно возрастать при большой неколлинеарности $\theta_{K} \rightarrow \pi / 4$ по сравнению со случаем коллинеарного ферромагнетика с $\theta_{K}=\pi / 2\left(\chi\left(\theta_{K}=\pi / 2\right)\right)$ (рис. 4).

\section{4. Фазовая диаграмма}

Зависимость поля спин-флоп-перехода от угла разворота осей анизотропии определяет границу между низкополевой А и высокополевой В фазами - фазовую диаграмму угол-поле (рис. 5). Асимптотические значения поля завершения переориентации фазовым переходом второго рода $h_{c}$ при $\theta_{K} \rightarrow \pi / 2$ соответствуют случаю коллинеарного ферромагнетика.

$$
h_{c}\left(\theta_{K}=\frac{\pi}{2}\right)=-2 a .
$$

При $\theta_{K} \rightarrow \pi / 4$ общая анизотропия исчезает, и разворот неколлинеарных моментов к направлению намагничивания происходит в сколь угодно малом поле. То же самое происходит в другом предельном случае одноосной легкоплоскостной анизотропии $a=0\left(K_{1}=K_{2}(1)\right)$.

На этой же диаграмме показана зависимость величины скачка относительной намагниченности при спин-флоп-

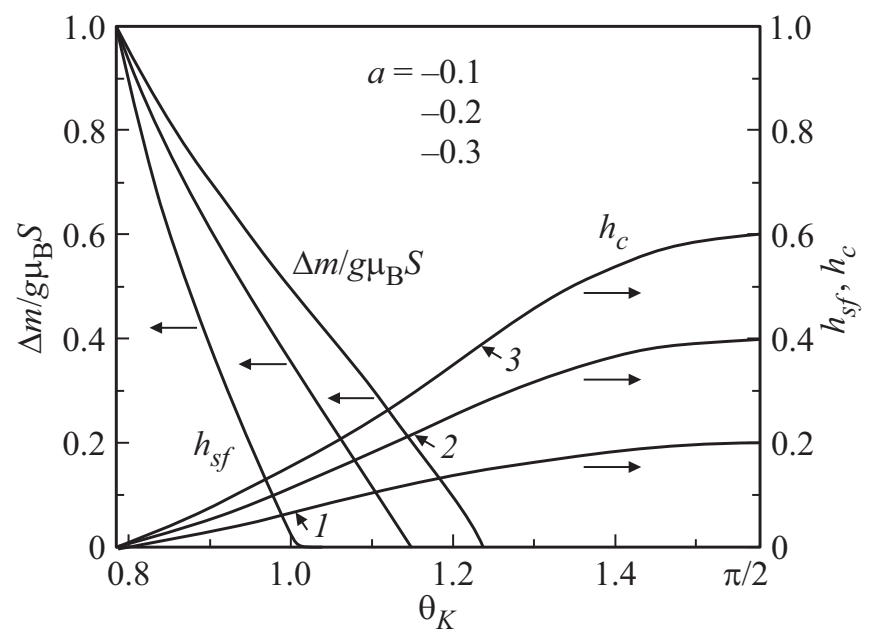

Рис. 5. Зависимость поля спин-флоп-перехода $h_{s f}$ (границы между фазами) и скачка относительной продольной намагниченности $\Delta m_{1} / g \mu_{B} S$ от угла ориентации оси анизотропии $\theta_{K}$ при параметре анизотропии $a=-0.1,-0.2,-0.3$. Цифрами 1 . 2 и 3 отмечены значения критического поля, выше которых фазовый переход становится переходом второго рода $h_{s f} \rightarrow h_{c}$ для величины локальной анизотропии $a=-0.1,-0.2,-0.3$, соответственно.

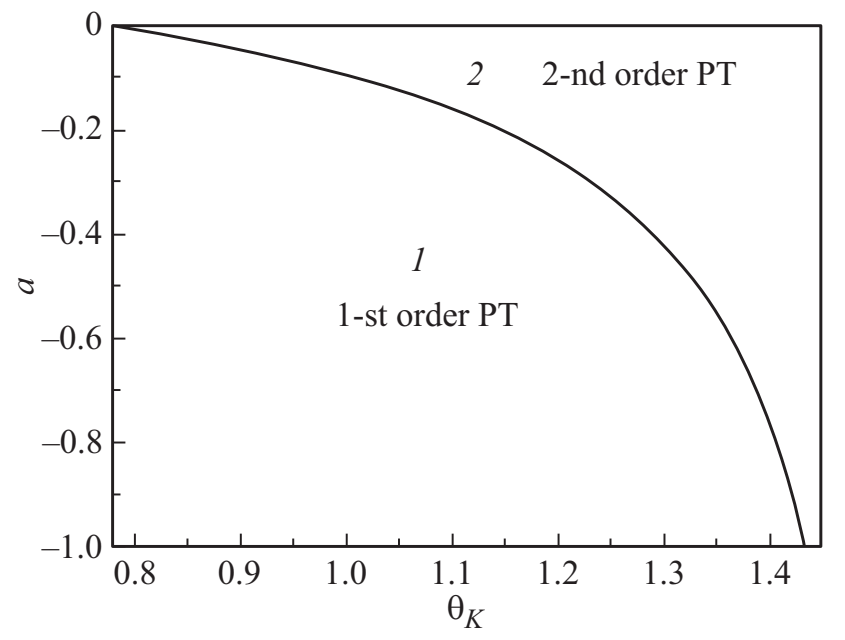

Рис. 6. Две области на плоскости параметров анизотропии с разным типом ориентационного фазового перехода. В области 1 (1-st order Phase Transition (PT)) переориентация заканчивается фазовым переходом первого рода. В области 2 (2-nd order PT) - переходом второго рода.

переходе от угла ориентации оси анизотропии. С ростом угла (приближении к коллинеарному случаю) скачок быстро убывает и в точках 1, 2 и 3 на кривых поля перехода для $a=-0.1,-0.2,-0.3$, соответственно, обращается в ноль. Выше этих значений ориентационный переход завершается фазовым переходом второго рода с критическим полем $h_{c}$.

Тип фазового перехода между состояниями (4) определяется параметрами анизотропии. Причем возникновение скачка продольной намагниченности при завершении ориентационного перехода из фазы А в фазу В носит пороговый характер по параметрам анизотропии $a$ и $\theta_{K}$. Соотношение между этими параметрами, при котором появляется скачок (переход становится переходом первого рода). можно получить из предельного условия существования фазы А

$$
\theta_{1}=-\theta_{2}=\theta_{c}
$$

в точке пересечения энергий фаз $\epsilon_{\mathrm{A}}$ и $\epsilon_{\mathrm{B}}$ (рис. $\left.2, b\right)$. При этом значение $\theta_{c}$ совпадает с углом $\theta$ фазы В. Кривые на рис. $2, b$ при предельном значении приложенного поля $h_{c}$ касаются друг друга

$$
\frac{d \epsilon_{\mathrm{A}}}{d h}=\frac{d \epsilon_{\mathrm{B}}}{d h}
$$

Выше этого значения поля решение системы (2) для фазы А отсутствует. Накладываемые условия при малой неколлинеарности подрешеток $\theta_{2}-\theta_{1} \ll 1$ (слабый антиферромагнетизм) приводят к значениям

$$
\sin 2 \theta_{c} \approx-a \sin 2 \theta_{K}, \quad h_{c} \approx 2 a \cos 2 \theta_{K} .
$$

Общее численное решение условий разделяет на плоскости параметров анизотропии области с разным типом фазового перехода (рис. 6). 
Необходимым условием завершения переориентации подрешеток в магнитном поле переходом первого рода является неколлинеарность магнитных моментов, вызванная одноионной анизотропией. Она приводит к появлению двух минимумов в угловой зависимости общей энергии анизотропии и, в результате, к двум минимумам в полевой зависимости энергии основного состояния (фазы А и В). Более быстрое изменение минимума фазы А приводит к совпадению значений минимумов фаз до их слияния. В этом случае пересечение энергий фаз происходит до завершения переориентации моментов фазы А (рис. $2, b)$ - ориентационный переход заканчивается фазовым переходом первого рода.

\section{5. Обсуждение}

В рассмотренной модели два параметра ОА: $a$ и $\theta_{K}$ определяют четыре значения, которые можно найти из экспериментальной кривой намагничивания: угол наклона кривой (начальная восприимчивость) (рис. 4), поле перехода, величину скачка намагниченности (рис. 5) и ширину гистерезиса магнитного поля (рис. 3). Труднее всего определить последние два значения. Учитывая возрастающую крутизну кривой при приближении поля к $h_{s f}$, необходимость точной ориентации кристалла относительно внешнего поля и сильную зависимость кривой намагничивания от температуры [19], точно определить величину скачка намагниченности сложно. Ширина гистерезиса может существенно зависеть от скорости прохождения точки перехода. Наиболее простым представляется определение параметров ОА из первых двух экспериментальных значений — начальной восприимчивости и поля перехода,

Магнитная анизотропия $\mathrm{PbMnBO}_{4}$ определяется сильным ян-теллеровским искажением кислородных октаэдров, окружающих ионы меди $M n^{3+}$ [19]. При этом длинная ось рассматривается как локальная легкая ось одноионной анизотропии. Искаженные октаэдры образуют цепочки вдоль оси $b$ с самым сильным ферромагнитным обменом между ионами марганца. Плоскости, содержащие длинные и короткие оси октаэдров, развернуты относительно общей легкой орторомбической оси кристалла $a$ на угол $\phi \approx 30^{0}$ (на рис. 1 показана проекция орторомбической оси $a$ ). Таким образом, энергия суммарной OА зависит от ориентации моментов четырех обменносвязанных трансляционно неэквивалентных подрешеток и, следовательно, определяется как локальной анизотропией. так и величинами обменных взаимодействий внутри и между цепочками. Эффективная константа не выражается через локальные простым суммированием последних, умноженных на функции углов $\theta_{K i}-$ эффективная ОА не является простой суммой каких-либо локальных проекций. Сравнение рассчитанных кривых намагничивания с экспериментально наблюдаемой зависимостью для $\mathrm{PbMnBO}_{4}$ [19] в поле, приложенном вдоль орторомбической оси $b$, дает параметры анизотропии $a \approx-0.1, \theta_{K} \approx 0.9$. Значение полученного угла ориентации локальной легкой оси близко к углу ориентации длинной диагонали искаженного кислородного октаэдра, окружающего ионы $\mathrm{Mn}^{3+}$, относительно оси $b \theta=0.84$. Для сравнения со значением величины ОА вдоль орторомбической оси $a$ (эффективной макроскопической анизотропии), определенной в рамках одноподрешеточной модели [23], воспользуемся значением обменного взаимодействия $J$, полученным в этой работе. Значение $a \approx-0.1$, полученное в настоящей работе в рамках двухподрешеточной модели (1), почти втрое превышает эффективную анизотропию [23]. Неколлинеарность локальных осей анизотропии многоподрешеточного ферромагнетика (как и антиферромагнетика [16]) не только приводит к характерному нелинейному виду кривой намагничивания со скачком магнитного момента, но и всегда дает меньшую величину наблюдаемой макроскопической анизотропии по сравнению с локальным значением, эффективно „усредняя“ анизотропию неколлинеарных подрешеток [11]. Детальный анализ анизотропных свойств $\mathrm{PbMnBO}_{4}$, включающий намагничивание вдоль самой трудной оси $c$, в рамках четырехподрешеточной модели с учетом ориентации осей октаэдров и влияния взаимодействия DM требует отдельного рассмотрения и будет представлен позднее. Отметим только, что совместное действие ОА и взаимодействия DM в этом магнетике сохраняет общий вид кривой намагничивания и спин-флоп-переход в поле, приложенном вдоль орторомбической оси $b$.

В настоящее время растущий интерес к изучению неколлинеарных магнетиков в значительной степени обусловлен магнитоэлектрическими свойствами, сопровождающими магнитную неколлинеарность [24,25]. Этот интерес вызван, прежде всего, возможностью управления магнитными параметрами кристалла, меняя электрическую поляризацию и наоборот, что имеет широкую технологическую перспективу. Идеальным магнитоэлектрическим мультиферроиком должен быть кристалл, в котором большая спонтанная поляризация была бы связана с большой намагниченностью [15]. При этом наиболее яркого проявления магнитоэлектрических свойств следует ожидать при скачкообразном изменении ориентации такой намагниченности в окресности фазового перехода первого рода. При таком переходе величина скачка намагниченности у слабого антиферромагнетика может быть значительно больше аналогичного скачка в слабом ферромагнетике. Немаловажным преимуществом является также значительно меньшее поле спин-флоп-перехода в слабом антиферромагнетике, определяемое только одноионной анизотропией. Отметим, что в изоструктурном кристалле $\mathrm{PbFeBO}_{4}$ обнаружены аномалии диэлектрических свойств при температуре установления ближнего и дальнего магнитного порядка, что указывает на корреляцию между магнитной и электрической подсистемами в этом кристалле [26]. 


\section{6. Заключение}

В ферромагнетике с неколлинеарными осями одноионной анизотропии магнитных подрешеток ориентационный фазовый переход в поле, приложенном в плоскости, содержащей локальные оси анизотропии, может происходить либо в виде двух фазовых переходов второго рода с непрерывным разворотом моментов подрешеток, либо в виде одного перехода второго рода и одного перехода первого рода. Во втором случае переход первого рода (спин-флоп-переход) сопровождается скачком проекции намагниченности на направление приложенного поля и гистерезисом. Переход имеет пороговый характер по параметрам анизотропии - величине локальной анизотропии и ориентации ее осей - и существует в широком интервале этих параметров. Получены зависимости поля спин-флоп-перехода, скачка намагниченности, начальной восприимчивости и границы появления фазового перехода первого рода от параметров локальной анизотропии подрешеток. Сравнение с экспериментальными кривыми намагниченности для $\mathrm{PbMnBO}_{4}$ позволили провести численную оценку параметров локальной анизотропии двухподрешеточной модели и сделать вывод об эффективном усреднении величины анизотропии при развороте осей.

\section{Благодарности}

Автор благодарен А.И.Панкрацу и А.Д.Балаеву за полезные обсуждения.

\section{Финансирование работы}

Работа выполнена при поддержке Российского Фонда Фундаментальных Исследований, Правительства Красноярского края, Красноярского Краевого Фонда Науки, исследовательский проект № 18-42-240008 „Влияние магнитной структуры на магнитодиэлектрические свойства оксидных кристаллов, содержащих стереоактивные ионы $\mathrm{Pb}^{2+}$ и $\mathrm{Bi}^{3+6}$.

\section{Конфликт интересов}

Автор заявляет, что у него нет конфликта интересов.

\section{Список литературы}

[1] S.V. Vonsovsky, E.A. Turov. J. Appl. Phys., 30, 9S, (1959).

[2] С.В. Вонсовский. Магнетизм, Наука, М. (1971). С. 754.

[3] Е.А. Туров. Физические свойства магнитоупорядоченных кристаллов. Изд. АН СССР, М. (1963). С. 177.

[4] I. Dzyaloshinsky. J. Phys. Chem. Solids 4, 241 (1958).

[5] T. Moriya. Phys. Rev. 120, 91 (1960).

[6] V.E. Dmitrienko, V. A. Chizhikov. Phys. Rev. Lett, 108, 187201 (2012)

[7] V. Yushankhai, M. Wolf, K.-H. Muller, R. Hayn, H. Rosner. Phys. Rev. B 62, 14229 (2000).

[8] T. Moriya. Phys. Rev. 117, 635 (1960).
[9] Дж. Смарт. Эффективное поле в теории магнетизма, Мир, М. (1968). C. 177.

[10] E.F. Bertaut. B cб.: Magnetism. Akademic Press, N. Y. (1963). V. 3, 149.

[11] С. Крупичка. Физика ферритов и родственных им магнитных окислов. Мир, М. (1976). Т. 2. С. 44.

[12] Е.А. Туров, А.В. Колчанов, В.В. Меньшенин, И.Ф. Мирсаев, В.В. Николаев. Симметрия и физические свойства антиферромагнетиков. Физматлит, М. (2001). С. 103.

[13] D. Coffey. J. Appl. Phys., 70, 6326, (1991).

[14] Г.А. Петраковский, М.А. Попов, А.Д. Балаев, К.А. Саблина, О.А. Баюков, Д.А. Великанов, А.М. Воротынов, А.Ф. Бовина, А.Д. Васильев, М. Боем. ФТТ 51, 1745 (2009).

[15] C. Weingart, N. Spaldin, E. Bousquet. Phys. Rev. B 86, 094413 (2012).

[16] С. Н. Мартынов. Письма в ЖЭТФ 108, 196 (2018).

[17] D. Talbayev, L. Mihaly, J. Zhou. Phys. Rev. Lett. 93, 017202 (2004).

[18] A.A. Mozhegorov, L.E. Gonchar, A. E. Nikiforov. Low Temp. Phys., 33, 229 (2007).

[19] A. Pankrats, K. Sablina, M. Eremin, A. Balaev, M. Kolkov, V. Tugarinov, A. Bovina. J. Magn. Magn. Mater, 414, 82 (2016).

[20] H. Park, J. Barbier. Acta Crystallog., E57, 82 (2001).

[21] H. Park, R. Lam, J. E. Greedan, J. Barbier. Chem. Matter. 15, 1703 (2003).

[22] Ю.А.Изюмов, В.Р. Сыромятников. Фазовые переходы и симметрия кристаллов. Наука, М. (1984). С. 105.

[23] A. Pankrats, M. Kolkov, S. Martynov, S. Popkov, A. Krasikov, A. Balaev, M. Gorev. J. Magn. Magn. Mater, 471, 416 (2019).

[24] T. Kimura, T. Goto, H. Shintani, K. Ishizaka, T. Arima, Y. Tokura. Nature (London) 426, 55 (2003).

[25] E. Bousquet, A. Cano, J. Phys: Condens. Matter 28, 1 (2016).

[26] A. Pankrats, K. Sablina, D. Velikanov, A. Vorotynov, O. Bayukov, A. Eremin, M. Molokeev, S. Popkov, A. Krasikov. J. Magn. Magn. Mater, 353, 23 (2014).

Редактор Т.Н. Василевская 